Draft version SePtember 10, 2021

Preprint typeset using $\mathrm{IATEX}_{\mathrm{E}} \mathrm{X}$ style emulateapj v. 11/26/04

\title{
CONSTRAINTS ON THE EXTRAGALACTIC BACKGROUND LIGHT FROM VERY HIGH ENERGY GAMMA-RAY OBSERVATIONS OF BLAZARS
}

\author{
Justin D. Finke, ${ }^{1}$ and Soebur Razzaque ${ }^{1}$ \\ U.S. Naval Research Laboratory, Code 7653, 4555 Overlook SW, Washington, DC, 20375-5352 \\ ${ }^{1} \mathrm{NRL} / \mathrm{NRC}$ Research Associate \\ Draft version September 10, 2021
}

\begin{abstract}
The extragalactic background light (EBL) from the infrared to the ultraviolet is difficult to measure directly, but can be constrained with a variety of methods. EBL photons absorb $\gamma$-rays from distant blazars, allowing one to use blazar spectra from atmospheric Cherenkov telescopes (ACTs) to put upper limits on the EBL by assuming a blazar source spectrum. Here we apply a simple technique, similar to the one developed by Schroedter (2005), to the most recent very-high energy (VHE) $\gamma$-ray observations of blazars to put upper limits on the EBL energy density. This technique is independent of the EBL model and has well-defined errors on the constraints. Our results are consistent with EBL measurements and constraints but marginally inconsistent with several EBL models.
\end{abstract}

Subject headings: gamma-rays: observations — BL Lacertae objects: general — diffuse radiation

\section{INTRODUCTION}

The EBL from the infrared through the visible and extending into the ultraviolet is thought to be dominated by direct starlight emission and absorption and re-radiation of starlight by dust. The EBL photons interact with VHE $\gamma$-rays from distant sources, such as blazars and gamma-ray bursts, producing $\mathrm{e}^{+}-\mathrm{e}^{-}$pairs and absorbing the VHE photons. Direct measurement of the EBL is difficult (see Hauser \& Dwek 2001, for a review) due to contamination by foreground zodiacal and Galactic light. Galaxy counts may also be used to estimate the EBL, but the unknown number of unresolved sources results in a lower limit. Many models have been developed (Salamon \& Stecker 1998; Malkan \& Stecker 1998, 2001; Stecker et al. 2006; Kneiske et al.|2002, 2004; Primack et al. 2001, 2005, 2008; Gilmore et al. 2008; Franceschini et al. 2008; Razzaque et al. 2009) but the input physics (e.g., star formation rate, dust absorption and re-emission) are not constrained enough to give a reliable answer.

It is possible to use VHE $\gamma$-ray observations of blazars from ACTs to constrain the EBL by assuming an intrinsic spectrum (Stecker \& de Jager 1993; Stanev \& Franceschini 1998). This was done by Aharonian et al. (2006a) using the hard observed spectrum of 1ES 1101-232 from HESS. They take a certain EBL shape (that of Aharonian et al. 2003b) that is consistent with EBL observations and lowered its normalization until they can fit the computed intrinsic spectrum with a power law softer than $\Gamma_{i n t}=1.5$. This gives an EBL quite close to the lower limits from galaxy counts. This technique has, however, been criticized because it assumes a particular shape of the EBL spectrum, which is not well known (Mazin \& Raue 2007). This criticism applies to several other constraints from blazar observations as well (e.g., Aharonian et al. 2007d). Mazin \& Raue (2007) have addressed this problem by developing a sophisticated technique which scans over a large grid of possible EBL shapes. They de-absorb blazar VHE $\gamma$ -

Electronic address: justin.finke@nrl.navy.mil ray spectra with every possible shape, and throw out the shapes which, when fit with a power-law, broken powerlaw, or power-law with an exponential cutoff, give $\Gamma_{\text {int }}$ greater than a certain value (they get two constraints, from $\Gamma_{\text {int }}>1.5$ and $\left.>0.75\right)$. The number of remaining allowed shapes give an upper limit on the EBL intensity. A similar technique was used by Krennrich et al. (2008) to rule out EBL models which are inconsistent with the recent lower limit at $3.6 \mu \mathrm{m}$ (Levenson \& Wright 2008). A relatively simple technique was developed by Schroedter (2005) to give upper limits on the EBL which is simpler than the technique of Mazin \& Raue (2007) and has well-defined errors. Schroedter (2005) applied his technique to the 6 blazars seen with ACTs at that time to get upper limits on the EBL energy density. To date, VHE $\gamma$-rays have been seen from 21 blazars, including 1 flat spectrum radio quasar (FSRQ) and $20 \mathrm{BL}$ Lac objects. In this paper, we apply the technique of Schroedter (2005) to an up-to-date sample of TeV blazar spectra.

Note that this technique, and other techniques to constrain the EBL with $\gamma$-ray observations from blazars do make assumptions about the intrinsic spectrum of blazars, and is thus not a limit in the conventional sense. That is, understanding of blazars' intrinsic spectra could improve in the future, which would change our conclusions. If blazars were found to typically have harder spectra than we assume here, our upper limits would be weaker, whereas if they were found to have typically softer spectra, they would be stronger.

In $\S 2$ we describe how one can put upper limits on the $\gamma \gamma$ absorption optical depth, $\tau_{\gamma \gamma}(\epsilon)$, and the EBL energy density, $\epsilon u_{E B L}(\epsilon ; z)$, using a method similar to the one developed by Schroedter (2005). In $\S 3$ we apply this technique to the most recent $\mathrm{ACT}$ spectra from blazars and discuss our results.

\section{CONSTRAINING THE EBL ENERGY DENSITY FROM GAMMA-RAY OBSERVATIONS}

The observed $\nu F_{\nu}$ spectrum of a distant source, $f_{o b s}$, at redshift $z$ is related to its unknown intrinsic spectrum, 
$f_{\text {int }}$ by

$$
f_{\text {obs }}(\epsilon)=e^{-\tau_{\gamma \gamma}(\epsilon)} f_{\text {int }}(\epsilon),
$$

where $\epsilon$ is the dimensionless observed $\gamma$-ray photon energy. The greater the absorption, the harder the intrinsic spectrum must be to produce a given observed spectrum. The hardest spectrum observed in the $\mathrm{TeV}$ range from a blazar near enough not to be strongly affected by EBL attenuation is $\Gamma \approx 1.9$ (where $\left.f(\epsilon)=f\left(\epsilon_{\min }\right)\left(\epsilon / \epsilon_{\text {min }}\right)^{-\Gamma+2}\right)$ from Mrk 501 (Aharonian et al. 1999). Two blazars observed with EGRET have $\Gamma \approx 1.5$, the FSRQ 0847120 and the TeV-observed high-peaked BL Lac Mrk 501 (Nandikotkur et al. 2007). The hardest blazar with the Large Area Telescope (LAT) in the Fermi GammaRay Space Telescope 3-month bright AGN source list is $\Gamma \sim 1.4$ from the BL Lac MS $1050.7+4946$ at $z=0.140$ (Abdo et al. 2009). This blazar has not been seen in the $\mathrm{TeV}$ range, however, and may be a different class of blazar. Just over half of the TeV blazars have been seen with Fermi and all of the TeV High-Peaked BL Lacs have Fermi spectral indices $\Gamma$ between 1.70 and 1.85. From a theoretical standpoint, shock-accelerated electrons are unlikely to produce $\gamma$-rays with spectral indices harder than $\Gamma=1.5$ from Compton scattering. We will therefore conservatively take $\Gamma_{i n t} \geq \Gamma_{i n t}^{\min }=1.5$ as the conventional limit on the spectral index. Several theoretical possibilities have been proposed to create harder spectra. Katarzyński et al. (2006) have suggested that electrons create synchrotron and Compton emission at a significant distance from the acceleration region, leading to a high cutoff in the lower portion of the electron spectrum that generates the $\mathrm{TeV} \gamma$-rays. This could result in harder observed spectra. Stecker et al. (2007) have performed simulations which generate harder electron spectra at the highest energies in relativistic shocks, and hence harder observed $\mathrm{TeV} \gamma$-ray spectra. Internal $\gamma \gamma$ absorption could possibly produce harder spectra in the TeV range (Aharonian et al. 2008b), although this would probably apply more to distant FSRQs with significant scattered radiation fields (i.e., broad line regions) which we do not consider in this paper. The decay of pions from a hadronic source could also produce an extremely hard $\mathrm{TeV}$ component, independent of the lower energy electron synchrotron emission (Mücke et al. 2003). Compton scattering of the cosmic microwave background radiation in the extended jets of blazars could produce harder spectra (Böttcher et al. 2008). These are possibilities that must be considered. Furthermore, creating a model consistent with the recent galaxy count result at $3.6 \mu \mathrm{m}$ by Levenson \& Wright (2008), Krennrich et al. (2008) find that spectral indices harder than $\Gamma_{\text {int }} \approx 1.5$ seem necessary. We will, therefore, take $\Gamma_{\text {int }} \leq \Gamma_{i n t}^{\text {min }}=1.0$ as the extreme limit.

By assuming the intrinsic spectrum is limited by $\Gamma_{i n t}^{\min }$ we can put an upper limit on $\tau_{\gamma \gamma}(\epsilon)$,

$$
\begin{gathered}
\tau_{\gamma \gamma}^{\max }(\epsilon, z)=\ln \left[\frac{f_{\text {int }}\left(\epsilon_{\min }\right)}{f_{\text {obs }}\left(\epsilon_{\min }\right)}\right]+ \\
\left(\Gamma_{\text {obs }}-\Gamma_{\text {int }}^{\min }\right) \ln \left(\epsilon / \epsilon_{\min }\right) .
\end{gathered}
$$

The standard error of $\tau_{\gamma \gamma}^{\max }$ is given by

$$
\begin{array}{r}
\sigma\left(\tau_{\gamma \gamma}^{\max }(\epsilon, z)\right)= \\
\sigma\left(f_{\text {obs }}(\epsilon)\right) / f_{\text {obs }}(\epsilon) .
\end{array}
$$

We use the $\gamma$-ray flux at $E_{\text {min }}=\epsilon_{\min } m_{e} c^{2} \mathrm{TeV}$, the lowest energy bin of the observation, to normalize the observed spectra. We then use the EBL model which gives the greatest $\tau_{\gamma \gamma}\left(\epsilon_{\text {min }}\right)$ (which is almost always the fast evolution model of Stecker et al. (2006) ) to de-absorb the spectra here, i.e., $f_{\text {int }}\left(\epsilon_{\min }\right)=f_{a b s}\left(\epsilon_{\min }\right) e^{\tau_{\gamma \gamma}\left(\epsilon_{\text {min }}\right)}$, and use this to normalize our intrinsic maximum spectra.

Once $\tau_{\gamma \gamma}^{\max }(\epsilon, z)$ is found, one can calculate an upper limit on the local EBL. This is done using several approximations, as follows. We begin with the $\gamma \gamma$ opacity of the universe as a function of the comoving EBL energy density, which is given by

$$
\begin{gathered}
\tau_{\gamma \gamma}\left(\epsilon_{1}, z\right)=\frac{c \pi r_{e}^{2}}{m_{e} c^{2} \epsilon_{1}^{2}} \int_{0}^{z} \frac{d z^{\prime}}{\left(1+z^{\prime}\right)^{2}}\left|\frac{d t_{*}}{d z^{\prime}}\right| \\
\int_{\frac{1}{\epsilon_{1}\left(1+z^{\prime}\right)}}^{\infty} d \epsilon^{\prime} \frac{u_{E B L}^{\prime}\left(\epsilon^{\prime} ; z^{\prime}\right)}{\epsilon^{\prime 3}} \bar{\phi}\left(\epsilon^{\prime} \epsilon_{1}\left(1+z^{\prime}\right)\right)
\end{gathered}
$$

where, in a flat $\Lambda$ CDM universe,

$$
\left|\frac{d t_{*}}{d z^{\prime}}\right|=\frac{1}{H_{0}\left(1+z^{\prime}\right) \sqrt{\Omega_{m}\left(1+z^{\prime}\right)^{3}+\Omega_{\Lambda}}},
$$

$u_{E B L}^{\prime}\left(\epsilon^{\prime} ; z^{\prime}\right)$ is the comoving EBL energy density per unit comoving dimensionless energy, $\epsilon^{\prime}=\epsilon(1+z)$,

$$
\begin{array}{r}
\bar{\phi}\left(s_{0}\right)=\frac{1+\beta_{0}^{2}}{1-\beta_{0}^{2}} \ln w_{0}-\beta_{0}^{2} \ln w_{0}-\frac{4 \beta_{0}}{1-\beta_{0}^{2}} \\
+2 \beta_{0}+4 \ln w_{0} \ln \left(1+w_{0}\right)-4 L\left(w_{0}\right), \\
\beta_{0}^{2}=1-1 / s_{0}, w_{0}=\left(1+\beta_{0}\right) /\left(1-\beta_{0}\right), \text { and } \\
L\left(w_{0}\right)=\int_{1}^{w_{0}} d w w^{-1} \ln (1+w)
\end{array}
$$

(Gould \& Schréder 1967; Brown et al. 1973).

If we assume the blazar is at low redshift, then $\left|d t_{*} / d z\right| \approx H_{0}^{-1}$, where we use $H_{0}=70 \mathrm{~km} \mathrm{~s}^{-1} \mathrm{Mpc}^{-1}$ for the Hubble constant. We also use the approximation that the EBL does not change significantly at low redshifts, and that all of the EBL photons are at the energy where the pair-production cross-section is largest, $\epsilon_{*}^{\prime}=2 /\left(\epsilon_{1}\left(1+z^{\prime}\right)\right) \approx 2 / \epsilon_{1}$ (i.e., we assume the EBL is monochromatic). The latter approximation overestimates the actual EBL energy density, since in reality the absorption will be spread out over a range of EBL photon energies, so that we are left with an upper limit on the EBL energy density, $\epsilon^{\prime} u_{E B L}^{\prime} \max _{B D}(\epsilon ; z)$. Using the Dirac delta-function these approximations are written as

$$
u_{E B L}^{\prime}\left(\epsilon^{\prime} ; z \approx 0\right) \approx \epsilon_{*}^{\prime} u_{E B L}^{\prime \max }\left(\epsilon_{*}^{\prime} ; z \approx 0\right) \delta\left(\epsilon^{\prime}-\epsilon_{*}^{\prime}\right) .
$$

Eqn. (8) allows us to perform the integrals in eqn. (4) so that

$$
\begin{array}{r}
\tau_{\gamma \gamma}^{\max }\left(\epsilon_{1}, z \approx 0\right)=\frac{c \pi r_{e}^{2} \epsilon_{1}}{8 m_{e} c^{2}} \\
\times \frac{\epsilon_{*}^{\prime} u_{E B L}^{\prime} \max _{*}\left(\epsilon_{*}^{\prime} ; z \approx 0\right)}{H_{0}} \bar{\phi}(2) z
\end{array}
$$

or

$$
\begin{aligned}
\epsilon_{*}^{\prime} u_{E B L}^{\prime} \max _{*}\left(\epsilon_{*}^{\prime} ; z \approx\right. & 0)=\frac{64 m_{e} c^{2} H_{0}}{3 c \sigma_{\mathrm{T}} z \bar{\phi}(2) \epsilon_{1}} \\
& \times \tau_{\gamma \gamma}^{\max }\left(2 / \epsilon_{*}^{\prime}, z \approx 0\right)
\end{aligned}
$$


where $\bar{\phi}(2) \approx 1.787$. The error in this EBL limit is given by

$$
\sigma\left(\epsilon_{*}^{\prime} u_{E B L}^{\prime \max }\right)=\frac{64 m_{e} c^{2} H_{0}}{3 c \sigma_{\mathrm{T}} \bar{\phi}(2) z \epsilon_{1}} \sigma\left(\tau_{\gamma \gamma}^{\max }\right)
$$

where $\sigma\left(\tau_{\gamma \gamma}^{\max }\right)$, the error in $\tau_{\gamma \gamma}^{\max }$, is given by eqn. (3). For $z \approx 0$, the comoving EBL energy density will be equal to the observed energy density, i.e.,

$$
\epsilon_{*} u_{E B L}\left(\epsilon_{*}, z \approx 0\right)=\epsilon_{*}^{\prime} u_{E B L}^{\prime}\left(\epsilon_{*}^{\prime}, z \approx 0\right) .
$$

For higher redshifts,

$$
\epsilon_{*} u_{E B L}\left(\epsilon_{*} ; z\right)=(1+z)^{-4} \epsilon_{*}^{\prime} u_{E B L}^{\prime}\left(\epsilon_{*}^{\prime} ; z\right) \text {. }
$$

The EBL energy density can be converted to intensity in units of, e.g., $\mathrm{nW} \mathrm{m}^{-2} \mathrm{sr}^{-1}$ by

$$
\epsilon I_{\epsilon}(z)=\frac{c}{4 \pi} \epsilon_{*} u_{E B L}\left(\epsilon_{*} ; z\right) .
$$

By inspecting eqns. (2) and (10) we see that $\epsilon u_{E B L}^{\max }(\epsilon ; z)$ will be minimized, thus giving the strongest constraint, if the dimensionless parameter

$$
\xi \equiv\left(\frac{T e V}{E_{\max }}\right) \frac{\Gamma_{o b s}-\Gamma_{i n t}^{\min }}{z} \ln \left(\frac{E_{\max }}{E_{\min }}\right)
$$

is minimized, where $E_{\max }=m_{e} c^{2} \epsilon_{\max }$ is the energy of the highest energy photon bin observed from a source. This parameter should be seen as a general guide. The highest energy photon bin is often not well observed, for example, in $\mathrm{H} 1426+428$ the highest energy bin is less than a $2 \sigma$ detection (Aharonian et al. 2003b).

\section{RESULTS AND DISCUSSION}

The blazar sample used to constrain the EBL is seen in Table 1. This is not a complete sample of TeV blazar spectra $^{1}$, as we have selected blazars that are nearby and should give the strongest constraints, i.e., that have the smallest $\xi$. The EBL upper limits from the 8 blazar spectra with the lowest $\xi$ are plotted in Fig. 1,

For each blazar, $i$, one gets a list of $\epsilon u_{E B L, j}^{\max }(\epsilon)$ at each EBL energy bin $j$, which corresponds to an observed $\gamma$-ray energy bin. For $N$ such blazars, we choose the $\min \left\{\epsilon u_{E B L, j}^{\max }(\epsilon)\right\}$ to give the strongest constraint on the $j$ th energy bin. These constraints, along with other EBL measurements and constraints, are plotted in Fig. 2.

In section $\S 2$ we have made several assumptions to get the upper limit, namely, that the EBL does not evolve at low redshift, and the absorption occurs entirely from an EBL photon of energy equal to the energy where the absorption cross-section is maximized. There is no direct way to measure the EBL at higher redshifts, however the star formation rate increases between $z=0.0$ and $z=0.2$ (e.g., Hopkins \& Beacom 2006) as does the luminosity density at various wavelengths (e.g., Le Floc'h et al. 2005; Babbedge et al. 2006). Thus it seems logical that the EBL energy density would be greater at higher redshifts. A greater EBL energy density in the past would result in a greater absorption, which would give weaker upper limits than what the actual limits would be if evolution was taken into account. Thus,

1 For complete, continually updated catalogs see the TeVCat (http://tevcat.uchicago.edu/) or R. Wagner's website (http://wWw.mppmu.mpg.de/ rwagner/sources/). if we did take evolution into account, our limits would be stronger. But the largest source of error is likely to be the monochromatic assumption. If the EBL energy density is significantly greater at higher energies (shorter wavelengths) than where the cross section is maximized $\left(\epsilon_{1} \epsilon^{\prime}=2\right)$, the result is a considerably weaker upper limit. In all of the EBL models presented in Fig. 2 the EBL is considerably higher at $\gtrsim 0.2 \mathrm{eV}(\lesssim 5 \mu \mathrm{m})$ than immediately below this energy. Thus, the EBL energy density at energies less than $0.2 \mathrm{eV}$ is likely to be considerably lower than our upper limits, although to actually quantify this error one would need to assume an EBL model. Since our conservative assumptions all result in upper limits erring on the high side, our constraints are quite strong.

These EBL upper limits may be compared to the constraints by Mazin \& Raue (2007). Their technique gives similar results and is in some ways complementary to our results. We use more constraining blazars than Mazin \& Raue (2007), particularly the blazar 1ES 0229+200, which gives a stronger constraint at $\sim 0.04 \mathrm{eV}(\sim 30 \mu \mathrm{m})$. The reported spectrum of 1ES 1101-232 (Aharonian et al. 2006a), which was used by Mazin \& Raue (2007), has been re-analyzed with improved energy calibration (Aharonian et al. 2007b), which we use instead. This is one reason our constraints are weaker than Mazin \& Raue (2007) in the $0.2-0.5 \mathrm{eV}$ $(3-7 \mu \mathrm{m})$ range. Mazin \& Raue (2007) also find that the size of the EBL grid to which they fit splines is a cause of systematic error. By using a finer grid, they find they can get constraints $20-30 \%$ higher. This could be another source of discrepancy between their results and ours, since our results do not make any assumptions as to the coarseness of the possible shape of the EBL spectrum. Additionally, our monochromatic assumption could lead to a higher upper limit, as discussed above. Mazin \& Raue (2007) estimate their errors in the optical and near-IR to be about 30\%. Our results are within these errors at $0.2-0.5 \mathrm{eV}$. Our results are lower in the optical $(\lesssim 10 \mu \mathrm{m}$ or $\gtrsim 1 \mathrm{eV})$ due to the assumption of the highest possible EBL energy density. They choose an arbitrary level larger than we do, since we choose the largest possible model, the fast evolution model of Stecker et al. (2006). The choice in this region does not act as a constraint, since our results will naturally be above the highest EBL model.

Our results are consistent with the recent highly constraining EBL lower limit at $0.34 \mathrm{eV}(3.6 \mu \mathrm{m})$ (Levenson \& Wright 2008) from number counts with the Spitzer Space Telescope (see Krennrich et al. 2008, for a different interpretation). The lower limit of Levenson \& Wright (2008) combined with our results and other data means that the EBL is quite well constrained at $3.6 \mu \mathrm{m}$. The only model above this limit is the best fit model of Kneiske et al. (2004). However, their model falls just above our conventional upper limit $\left(\Gamma_{\text {int }}^{\min }=1.5\right)$ at $\approx 0.7 \mathrm{eV}(\approx 1.7 \mu \mathrm{m})$. The models of Gilmore et al. (2008), Franceschini et al. (2008), and the fast evolution model of Stecker et al. (2006) also fall above our conventional limit at $\approx 0.04 \mathrm{eV}(\approx 30 \mu \mathrm{m})$, while the baseline model of Stecker et al. (2006) is just below our upper limit. None of the models are above the extreme limit $\left(\Gamma_{i n t}^{\min }=1\right)$, which is not surprising, considering this weaker limitation was designed to avoid con- 

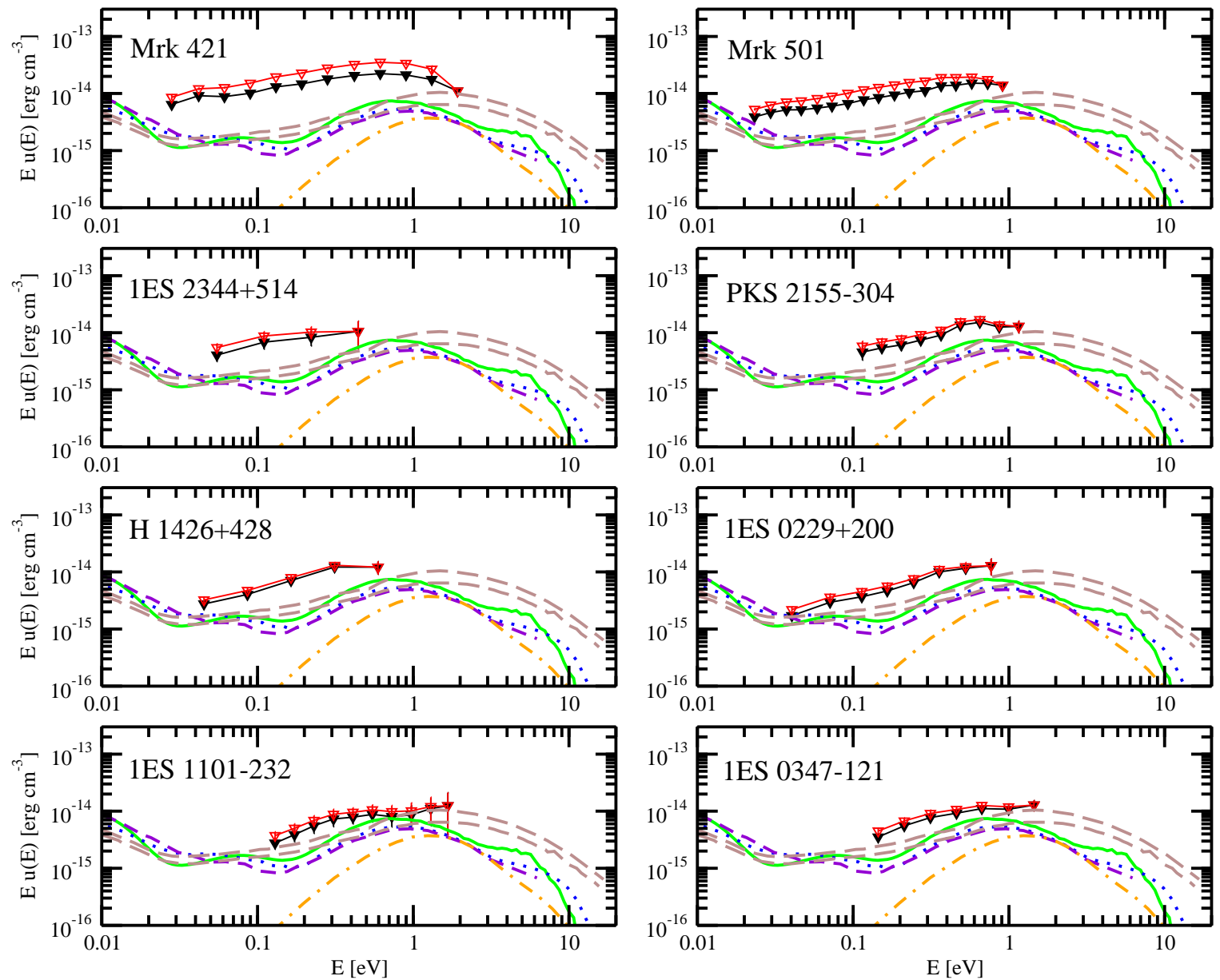

FIG. 1.- Upper limits for the conventional $\left(\Gamma_{i n t}^{m i n}=1.5\right.$; black filled inverted triangles $)$ and extreme $\left(\Gamma_{i n t}^{m i n}=1.0\right.$; red empty inverted triangles) limits on the EBL. Also plotted are several EBL models: the best fit model from Kneiske et al. (2004, solid green curve), the fiducial model from Gilmore et al. (2008, dotted blue curve), the model of Franceschini et al. (2008, short dashed violet curve), the baseline and fast evolution models of Stecker et al. (2006, lower and upper long dashed brown curves, respectively), and model B from Razzaque et al. (2009, dot-dashed orange curve).

flicts between $\gamma$-ray observations and EBL models. Future observations of blazars with ACTs as well as the recently launched Fermi Gamma-Ray Space Telescope will provide further upper limits on EBL models.

We have updated the technique of Schroedter (2005) to a recent sample of VHE $\gamma$-ray blazar spectra to obtain upper limits on the EBL energy density. This technique does not make assumptions about the shape of the EBL or fit a certain model to a de-absorbed spectrum, thus avoiding problems with assuming an EBL spectral shape in other techniques for using $\mathrm{VHE} \gamma$-ray observations to constrain the EBL. We obtain upper limits weaker and more conservative than limits from de-absorbing spectra with an assumed EBL shape and fitting the results with a power-law (e.g., Aharonian et al. 2006a) and in agreement with a similar technique by Mazin \& Raue (2007). Our conservative assumptions imply that it is likely the actual EBL energy density is considerably lower than our upper limits, especially for $m_{e} c^{2} \epsilon \lesssim 0.2 \mathrm{eV}$.

We thank the anonymous referee and C. Dermer for useful comments which have improved this paper. S.R. is supported by the National Research Council associateship program at the Naval Research Laboratory. J.D.F. was supported by NASA Swift Guest Investigator Grant DPR-NNG05ED411 and NASA GLAST Science Investigation DPR-S-1563-Y.

\section{REFERENCES}

Abdo, A. A. et al. 2009, ArXiv e-prints

Acciari, V. A. et al. 2008, ApJ, 684, L73

-. 2009, ArXiv e-prints

Aharonian, F. et al. 2003a, A\&A, 406, L9

-. 2003b, A\&A, 403, 523

-. 2005a, A\&A, 436, L17

-. 2005b, A\&A, 430, 865

-. 2006a, Nature, 440, 1018

-. 2006b, A\&A, 455, 461

-. 2007a, ApJ, 664, L71

-. 2007b, A\&A, 470, 475

-. 2007c, A\&A, 473, L25
-. 2007d, A\&A, 475, L9

-. 2008a, A\&A, 481, L103

Aharonian, F. A., Khangulyan, D., \& Costamante, L. 2008b, MNRAS, 387, 1206

Aharonian, F. A. et al. 1999, A\&A, 349, 11

Albert, J. et al. 2006a, ApJ, 642, L119

-. 2006b, ApJ, 648, L105

-. 2007a, ApJ, 666, L17

-. 2007b, ApJ, 667, L21

Babbedge, T. S. R. et al. 2006, MNRAS, 370, 1159

Bernstein, R. A., Freedman, W. L., \& Madore, B. F. 2002, ApJ, 571,56 
TABLE 1

TeV Blazar Sample

\begin{tabular}{|c|c|c|c|c|c|c|}
\hline Blazar & Redshift & Spectral Index $(\Gamma)$ & $E_{\min }[\mathrm{TeV}]$ & $E_{\max }[\mathrm{TeV}]$ & $\xi\left(\Gamma_{i n t}^{\min }=1.5\right)$ & Reference \\
\hline Mrk 421 & 0.030 & $2.14 \pm 0.10$ & 0.3 & 18 & 5.7 & Krennrich et al. (2001) \\
\hline Mrk 421 & 0.030 & $2.09 \pm 0.09$ & 0.4 & 8 & 8.6 & Krennrich et al. (2002) \\
\hline Mrk 501 & 0.034 & $1.92 \pm 0.15$ & 0.6 & 22 & 2.6 & Aharonian et al. (1999) \\
\hline $1 \mathrm{ES} 2344+514$ & 0.044 & $2.54 \pm 0.18$ & 1.2 & 11 & 8.6 & Schroedter et al. (2005) \\
\hline Mrk 180 & 0.045 & $3.3 \pm 0.7$ & 0.2 & 4.2 & 29.0 & Albert et al. (2006b) \\
\hline $1 \mathrm{ES} 1959+650$ & 0.047 & $3.18 \pm 0.17$ & 1.5 & 11 & 9.1 & Aharonian et al. (2003a) \\
\hline BL Lacertae & 0.069 & $3.64 \pm 0.54$ & 0.2 & 0.7 & 55.5 & Albert et al. (2007a) \\
\hline PKS 2005-489 & 0.071 & $4.0 \pm 0.4$ & 0.2 & 2.5 & 35.6 & Aharonian et al. (2005a) \\
\hline RGB $0152+017$ & 0.080 & $2.95 \pm 0.41$ & 0.3 & 3.0 & 16.4 & Aharonian et al. (2008a) \\
\hline W Comae & 0.102 & $3.81 \pm 0.35$ & 0.3 & 1.1 & 35.1 & Acciari et al. (2008) \\
\hline PKS 2155-304 & 0.116 & $3.19 \pm 0.17$ & 0.2 & 5 & 9.4 & Aharonian et al. (2007a) \\
\hline PKS 2155-304 & 0.116 & $2.84 \pm 0.24$ & 0.4 & 4 & 8.5 & Aharonian et al. (2005b) \\
\hline H $1426+428$ & 0.129 & $2.60 \pm 0.60$ & 0.8 & 10 & 3.3 & Aharonian et al. (2003b) \\
\hline $1 \mathrm{ES} 0229+200$ & 0.139 & $2.50 \pm 0.21$ & 0.6 & 12 & 2.5 & Aharonian et al. (2007d) \\
\hline H 2356-309 & 0.165 & $3.09 \pm 0.26$ & 0.2 & 1 & 15.5 & Aharonian et al. (2006b) \\
\hline $1 \mathrm{ES} 1218+304$ & 0.182 & $3.00 \pm 0.40$ & 0.08 & 0.7 & 14.7 & Albert et al. (2006a) \\
\hline $1 \mathrm{ES} 1218+304$ & 0.182 & $3.08 \pm 0.41$ & 0.2 & 1.4 & 12.1 & Acciari et al. (2009) \\
\hline 1ES $1101-232$ & 0.186 & $2.94 \pm 0.20$ & 0.3 & 3.3 & 6.6 & Aharonian et al. (2007b) \\
\hline $1 \mathrm{ES} 0347-121$ & 0.188 & $3.10 \pm 0.25$ & 0.3 & 3.0 & 7.7 & Aharonian et al. (2007c) \\
\hline $1 \mathrm{ES} 1011+496$ & 0.212 & $4.0 \pm 0.5$ & 0.2 & 0.6 & 21.6 & Albert et al. (2007b) \\
\hline
\end{tabular}

Böttcher, M., Dermer, C. D., \& Finke, J. D. 2008, ApJ, 679, L9

Brown, R. W., Mikaelian, K. O., \& Gould, R. J. 1973, Astrophys. Lett., 14, 203

Cambrésy, L., Reach, W. T., Beichman, C. A., \& p Jarrett, T. H. 2001, ApJ, 555, 563

Dole, H., Lagache, G., Puget, J.-L., Caputi, K. I., FernándezConde, N., Le Floc'h, E., Papovich, C., Pérez-González, P. G., Rieke, G. H., \& Blaylock, M. 2006, A\&A, 451, 417

Dwek, E. \& Arendt, R. G. 1998, ApJ, 508, L9

Fazio, G. G. et al. 2004, ApJS, 154, 39

Franceschini, A., Rodighiero, G., \& Vaccari, M. 2008, A\&A, 487, 837

Gilmore, R. C., Madau, P., Primack, J. R., \& Somerville, R. S. 2008, in American Institute of Physics Conference Series, Vol. 1085, American Institute of Physics Conference Series, 577-580

Gorjian, V., Wright, E. L., \& Chary, R. R. 2000, ApJ, 536, 550

Gould, R. J. \& Schréder, G. P. 1967, Physical Review, 155, 1404

Hauser, M. G. \& Dwek, E. 2001, ARA\&A, 39, 249

Hauser, M. G. et al. 1998, ApJ, 508, 25

Hopkins, A. M. \& Beacom, J. F. 2006, ApJ, 651, 142

Katarzyński, K., Ghisellini, G., Tavecchio, F., Gracia, J., \& Maraschi, L. 2006, MNRAS, 368, L52

Kneiske, T. M., Bretz, T., Mannheim, K., \& Hartmann, D. H. 2004, A\&A, 413, 807

Kneiske, T. M., Mannheim, K., \& Hartmann, D. H. 2002, A\&A, 386,1

Krennrich, F., Dwek, E., \& Imran, A. 2008, ApJ, 689, L93

Krennrich, F. et al. 2001, ApJ, 560, L45

-. 2002, ApJ, 575, L9

Le Floc'h, E. et al. 2005, ApJ, 632, 169

Levenson, L. R. \& Wright, E. L. 2008, ApJ, 683, 585

Levenson, L. R., Wright, E. L., \& Johnson, B. D. 2007, ApJ, 666, 34

Madau, P. \& Pozzetti, L. 2000, MNRAS, 312, L9
Malkan, M. A. \& Stecker, F. W. 1998, ApJ, 496, 13

-. 2001, ApJ, 555, 641

Mazin, D. \& Raue, M. 2007, A\&A, 471, 439

Metcalfe, L. et al. 2003, A\&A, 407, 791

Mücke, A., Protheroe, R. J., Engel, R., Rachen, J. P., \& Stanev, T. 2003, Astroparticle Physics, 18, 593

Nandikotkur, G., Jahoda, K. M., Hartman, R. C., Mukherjee, R., Sreekumar, P., Böttcher, M., Sambruna, R. M., \& Swank, J. H. 2007, ApJ, 657, 706

Papovich, C. et al. 2004, ApJS, 154, 70

Primack, J. R., Bullock, J. S., \& Somerville, R. S. 2005, in American Institute of Physics Conference Series, Vol. 745, High Energy Gamma-Ray Astronomy, ed. F. A. Aharonian, H. J. Völk, \& D. Horns, 23-33

Primack, J. R., Gilmore, R. C., \& Somerville, R. S. 2008, in American Institute of Physics Conference Series, Vol. 1085, American Institute of Physics Conference Series, 71-82

Primack, J. R., Somerville, R. S., Bullock, J. S., \& Devriendt, J. E. G. 2001, in American Institute of Physics Conference Series, Vol. 558, American Institute of Physics Conference Series, ed. F. A. Aharonian \& H. J. Völk, 463-+

Razzaque, S., Dermer, C. D., \& Finke, J. D. 2009, ApJ, in press, arXiv:0807.4294

Salamon, M. H. \& Stecker, F. W. 1998, ApJ, 493, 547

Schroedter, M. 2005, ApJ, 628, 617

Schroedter, M. et al. 2005, ApJ, 634, 947

Stanev, T. \& Franceschini, A. 1998, ApJ, 494, L159+

Stecker, F. W., Baring, M. G., \& Summerlin, E. J. 2007, ApJ, 667, L29

Stecker, F. W. \& de Jager, O. C. 1993, ApJ, 415, L71+

Stecker, F. W., Malkan, M. A., \& Scully, S. T. 2006, ApJ, 648, 774

Wright, E. L. \& Reese, E. D. 2000, ApJ, 545, 43 


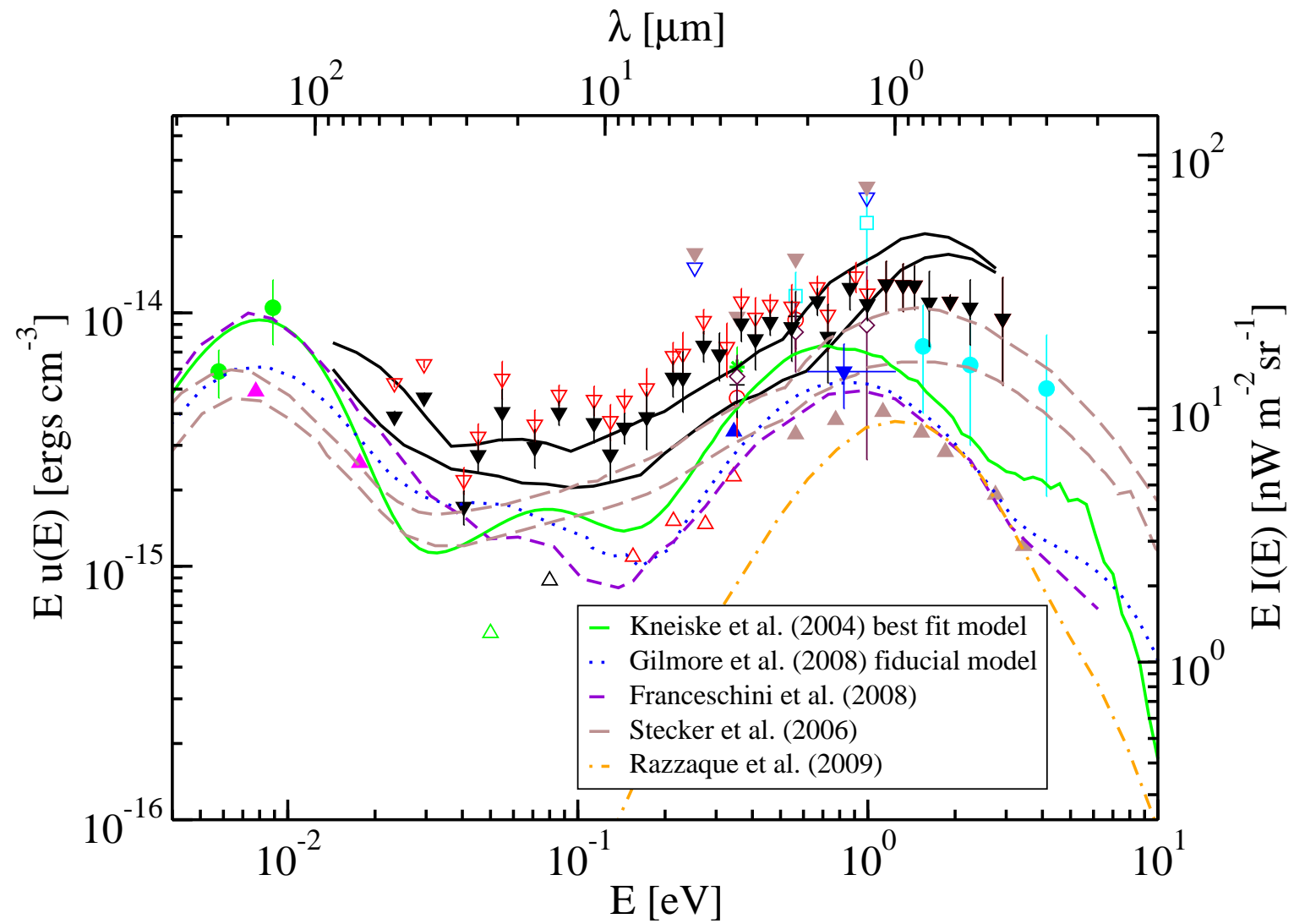

FIG. 2.- Measurements and constraints on the EBL energy density from observations. Measurements are from Bernstein et al. (2002, cyan points), Goriian et al. (2000, empty red circle), Dwek \& Arendt (1998, green asterisk), Cambrésv et al. (2001, empty cyan square), Wright \& Reese (2000, black cross), Levenson et al. (2007, maroon diamonds), and Hauser et al. (1998, green filled circles). Lower limits are from Fazio et al. (2004, red empty triangles), Madau \& Pozzetti (2000, brown filled triangles), Levenson \& Wright (2008, blue filled triangle), Dole et al. (2006, magenta filled triangles), Metcalfe et al. (2003, black empty triangle), and Papovich et al. (2004, green empty triangle). Upper limits are from Hauser et al. (1998, brown filled inverted triangles), Dwek \& Arendt (1998, blue empty inverted triangles), Aharonian et al. (2006a, blue filled inverted triangle), Mazin \& Raue (2007, upper and lower black curves $\Gamma_{i n t}^{m i n}=0.75$ and $\Gamma_{i n t}^{m i n}=1.5$ upper limits, respectively), and Red empty and black filled inverted triangles are the $\Gamma_{i n t}^{\min }=1.0$ and $\Gamma_{i n t}^{\min }=1.5$ upper limits, respectively, from this paper. The curves are the same models as in Fig. 1 